ARTICLE

\title{
Adrenergic stress constrains the development of anti-tumor immunity and abscopal responses following local radiation
}

Minhui Chen', Guanxi Qiao', Bonnie L. Hylander ${ }^{1}$, Hemn Mohammadpour ${ }^{1}$, Xiang-Yang Wang ${ }^{2}$, John R. Subjeck ${ }^{3}$, Anurag K. Singh ${ }^{3,4} \&$ Elizabeth A. Repasky ${ }^{1 凶}$

The abscopal effect following ionizing radiation therapy (RT) is considered to be a rare event. This effect does occur more frequently when combined with other therapies, including immunotherapy. Here we demonstrate that the frequency of abscopal events following RT alone is highly dependent upon the degree of adrenergic stress in the tumor-bearing host. Using a combination of physiologic, pharmacologic and genetic strategies, we observe improvements in the control of both irradiated and non-irradiated distant tumors, including metastatic tumors, when adrenergic stress or signaling through $\beta$-adrenergic receptor is reduced. Further, we observe cellular and molecular evidence of improved, antigen-specific, anti-tumor immune responses which also depend upon $T$ cell egress from draining lymph nodes. These data suggest that blockade of $\beta 2$ adrenergic stress signaling could be a useful, safe, and feasible strategy to improve efficacy in cancer patients undergoing radiation therapy.

\footnotetext{
${ }^{1}$ Department of Immunology, Roswell Park Comprehensive Cancer Center, Elm and Carlton Streets, Buffalo, NY 14263, USA. ${ }^{2}$ Department of Genetics, Virginia Commonwealth University, Richmond, VI 23298, USA. ${ }^{3}$ Department of Cell Stress Biology, Roswell Park Comprehensive Cancer Center, Elm and Carlton Streets, Buffalo, NY 14263, USA. ${ }^{4}$ Department of Radiation Oncology, Roswell Park Comprehensive Cancer Center, Elm and Carlton Streets, Buffalo,

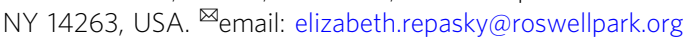


R adiotherapy is one of the most common treatments for cancer. While its primary mechanism of action is thought to be direct damage to tumor cells in the field of irradiation $^{1}$, it has been reported occasionally that some patients treated with local ionizing radiation therapy (RT), experience a regression of distant, non-irradiated tumor $(s)^{2}$. This intriguing phenomenon, referred to as the "abscopal effect" 3,4 , is now thought to be due to the ability of RT to stimulate a systemic anti-tumor immune responses ${ }^{5-8}$. Indeed, as early as the 1970s, investigators conducting research on the efficacy of RT in mouse tumor models observed reduced efficacy in immunodeficient nude mice compared to immunocompetent mice ${ }^{9}$ pointing to a role for the adaptive immune response in the efficacy of RT. However, even in immunocompetent mice, it is clear that the abscopal response is disappointingly rare and unpredictable unless radiation is combined with immunotherapy ${ }^{10-18}$ or other therapies ${ }^{19,20}$. A more complete understanding of the interaction of RT and the immune response in pre-clinical models could reveal new strategies to increase the frequency of abscopal effects in the clinic, resulting in improved patient outcomes ${ }^{21}$.

Relative to this problem, during pre-clinical research using murine models of cancer and graft versus host disease, our lab has clearly demonstrated that Institutional Animal Care and Use Committee (IACUC) - mandated mild cool housing temperature for laboratory mice (typically $\sim 22-23^{\circ} \mathrm{C}$ ) is sufficient to cause chronic adrenergic stress ${ }^{22}$ with activation of sympathetic nerves resulting in norepinephrine release needed for adaptive thermogenesis $^{23-25}$. That this has a direct impact on the outcomes of studies involving a variety of disease models, including studies involving immune responses has been shown by comparing outcomes in mice housed under standard vs. thermoneutral temperatures ${ }^{24-27}$. Furthermore, our lab discovered that this chronic stress suppresses baseline anti-tumor immunity and accelerates tumor growth and metastasis ${ }^{28,29}$, while it also suppresses graft versus host disease following allogeneic hematopoietic stem cell transplantation ${ }^{30,31}$. When tumor-bearing mice are housed at their thermoneutral temperature $\left(\sim 30^{\circ} \mathrm{C}\right)$, chronic adrenergic stress and norepinephrine production are significantly lowered, $\mathrm{CD}^{+} \mathrm{T}$ cell-dependent responses are significantly improved, while numbers and activities of immunosuppressive cells are reduced ${ }^{29,32,33}$. Alternatively, when $\beta 2$ - adrenergic receptor (AR) knockout (KO) mice were used, or if wildtype (WT) mice housed at $22^{\circ} \mathrm{C}$ were given the pan- $\beta$-AR blocker propranolol, we observed a similar improvement in tumor growth control and increased $\mathrm{CD} 8^{+} \mathrm{T}$ cell infiltration was seen in mice housed at $30^{\circ} \mathrm{C}^{22,29,34}$, suggesting that cool housing-induced adrenergic stress exerts its negative effects on the anti-tumor immune system through activation of $\beta$-ARs on immune cells.

We, and others, have also shown that adrenergic signaling directly increases tumor cell resistance to killing by chemotherapy and targeted therapies $22,35,36$, and our lab recently showed that standard housing temperature-induced adrenergic stress signaling increases intrinsic tumor cell resistance and constrains the antitumor efficacy of ionizing radiation ${ }^{37}$. However, neither we nor others have ever explored the relationship between the frequency of abscopal effects and housing temperature, or the role of host adrenergic stress signaling in general on anti-tumor immunity following RT. Here we use three different tumor models as well as physiological, pharmacological, and genetic strategies that reduce or block $\beta 2$-adrenergic signaling to examine the impact of adrenergic stress signaling on the frequency of the radiation-induced abscopal effect. Our data reveal a surprising dependency of the overall efficacy of RT (against both irradiated and non-irradiated, distant tumors) on baseline chronic adrenergic stress due to housing temperature, and more specifically, to $\beta 2-\mathrm{AR}$ signaling. These data provide a compelling rationale for testing RT in combination with $\beta$-blockers and/or other stress reducing strategies in cancer patients undergoing RT.

\section{Results}

Adrenergic stress blunts efficacy of RT and abscopal effects. We previously reported that the standard cool housing temperature mandated for laboratory mice accelerates tumor growth and metastasis and suppresses anti-tumor immunity by subjecting mice to chronic adrenergic stress ${ }^{28,29}$. Here, we investigated the effect of adrenergic stress on the efficacy of local RT in three different tumor types (CT26.CL25 colon tumors in BALB/ cAnNcr $(\mathrm{BALB} / \mathrm{c})$ mice and B16 melanoma in $\mathrm{C} 57 \mathrm{BL} / 6 \mathrm{NCr}$ (C57BL/6) mice, and 4T1 mammary tumors in BALB/c mice-a clinically relevant metastatic model). We also used three different strategies for manipulating adrenergic stress: physiological, pharmacological, and genetic. We began by testing whether the efficacy of ionizing radiation and, specifically, the abscopal effect $^{5,8,38}$, is influenced by the physiological stress induced by housing mice at the standard (IACUC-mandated) housing temperature. As shown in Fig. 1a, we maintained mice at either $22^{\circ} \mathrm{C}$ (the standard housing temperature, ST) used at Roswell Park or acclimated them to $30^{\circ} \mathrm{C}$ (the thermoneutral temperature, TT, for laboratory mice which alleviates cold stress and results in reduced levels of norepinephrine (NE)) for at least 3 weeks. We then implanted either CT26.CL25 colon tumor cells (hereafter referred to as CT26) or B16 melanoma cells in both hind limbs. When the tumors became palpable, one tumor in each mouse was exposed to local RT (6 Gy for CT26, 20 Gy for B16). We selected these radiation doses for this study as they produce a reproducible slowing of tumor growth but are suboptimal for each tumor type and do not result in complete tumor control nor in abscopal effects. As we have shown previously, tumors in mice housed at ST grew significantly faster than those housed at TT (which reduces adrenergic stress). Indeed, the tumor growth inhibition seen by simply housing mice at TT was similar to that achieved by treatment of mice at ST with radiation; the effect of radiation was further enhanced when mice were housed at TT (CT26, Fig. 1a left; B16, Supplementary Fig. 1a left). By comparison, in mice housed at ST, RT had no effect on the contralateral, nonirradiated tumor, while there was a reduced growth rate in those tumors in all of the mice housed at TT mice, i.e., an abscopal effect was generated (CT26, Fig. 1a right and Supplementary Fig. 1c left; B16, Supplementary Fig. 1a right and b). That this abscopal effect is mediated by immune activity is supported by the presence of an increased number of intratumoral CD8 ${ }^{+}$ $\mathrm{T}$ cells, especially $\mathrm{CD} 8^{+}$effector/memory T cells (Supplementary Fig. 1c middle and right) in the contralateral, non-irradiated tumors of mice housed at TT.

Since we know that housing at $22^{\circ} \mathrm{C}$ increases adrenergic stress, we next used a pharmacological strategy, treatment of mice with the $\beta$-AR antagonist propranolol (Prop), to test the role of $\beta$ ARs in response to radiation. This experiment had four groups of mice at $22^{\circ} \mathrm{C}$ : (1) phosphate-buffered saline (PBS) alone, (2) Prop alone, (3) PBS + RT, and (4) Prop + RT. We found that compared to PBS and RT alone, Prop plus RT slowed tumor growth of both the irradiated and contralateral, non-irradiated tumors (Fig. 1b), indicating that a blockade of adrenergic signaling greatly improves efficacy of RT in mice housed at ST. We repeated this experiment with SCID (C.B. Igh-1b Icr Tac Prkdc scid) mice, which are characterized by an absence of functional $\mathrm{T}$ and $\mathrm{B}$ cells, and neither radiation nor Prop had any effect on tumor growth of the irradiated tumor or the contralateral, non-irradiated tumor (Fig. 1c), indicating that the overall effect of this dose of radiation on both the primary and contralateral tumor depends upon an intact immune system. 


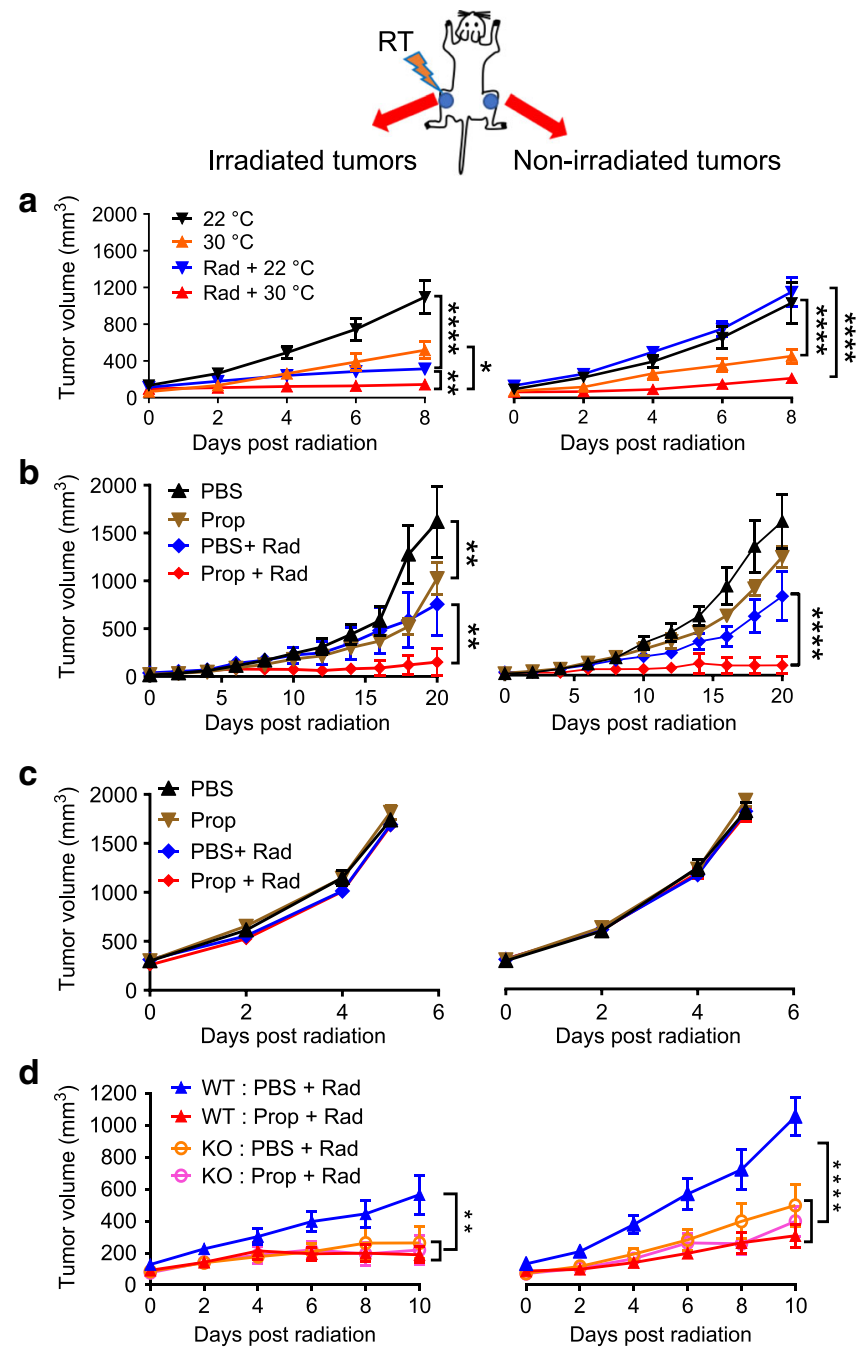

Fig. 1 Reduction in $\boldsymbol{\beta 2}$-adrenergic signaling enhances the abscopal effect in a CT26 colon carcinoma model. BALB/c mice were implanted with CT26 tumors bilaterally; one tumor was irradiated. Tumor growth is shown for irradiated tumors (left) and non-irradiated tumors (right). a In mice housed at $22^{\circ} \mathrm{C}$ (ST) or $30^{\circ} \mathrm{C}$ (TT); $\mathbf{b}$ and $\mathbf{c}$ Comparison of tumor growth in BALB/C b or SCID mice $\mathbf{c}$, which received no treatment, propranolol alone, radiation alone, or the combination; $\mathbf{d}$ Comparison of tumor growth in WT or $\beta 2$-AR $\mathrm{KO}$ mice which received no treatment, propranolol alone, radiation alone, or the combination. Data are presented as mean \pm SEM. ${ }^{\star} P<0.05$; ${ }^{\star \star} P<0.01$; ${ }^{\star \star \star \star} P<0.0001$ (two-way ANOVA analysis). For $\mathbf{a}, n=9$ biologically independent mice in $22^{\circ} \mathrm{C}, 30^{\circ} \mathrm{C}$ and $\operatorname{Rad}+30^{\circ} \mathrm{C}$ groups, $n=7$ biologically independent mice in $\mathrm{Rad}+22^{\circ} \mathrm{C}$ group; for $\mathbf{b}, n=6$ biologically independent mice in PBS, Prop, and PBS + Rad groups, $n=5$ biologically independent mice in Prop + Rad group; for $\mathbf{C}, n=10$ biologically independent mice in all groups; for $\mathbf{d}, n=6$ biologically independent mice in WT PBS + Rad and KO PBS + Rad groups, $n=7$ biologically independent mice in WT Prop + Rad group, $n=8$ biologically independent mice in KO Prop + Rad group.

To further dissect the role of $\beta$-adrenergic signaling in the efficacy of RT, we utilized a genetic strategy with $\beta 2$-AR KO mice and found that the growth of CT26 irradiated and contralateral non-irradiated tumors $\beta 2$-AR KO mice with or without Prop, was similar to that seen in irradiated WT mice given Prop (Fig. 1d), suggesting that the improved efficacy and abscopal effect is dependent on $\beta 2$-ARs. Taken together, these data support the idea that adrenergic signaling is a critical pathway by which the efficacy of RT against both irradiated and non-irradiated distant tumors is regulated.

Impact of reducing $\beta$-AR signaling depends on $\mathrm{CD8}^{+} \mathrm{T}$ cells. Data obtained from SCID mice (Fig. 1c) strongly suggest that the anti-tumor immune response is a major target of reducing or blocking adrenergic signaling in combination with RT. Supporting this assumption, depletion of $\mathrm{CD} 8^{+} \mathrm{T}$ cells in $\mathrm{BALB} / \mathrm{c}$ mice treated with radiation and Prop greatly reduced the improved efficacy seen against both irradiated and distant non-irradiated tumors (Fig. 2a). Moreover, $\mathrm{CD}^{+} \mathrm{T}$ cell depletion also eliminated the improved tumor control seen in irradiated $\beta 2$-AR KO mice (Fig. 2b). These data suggest that $\mathrm{CD}^{+} \mathrm{T}$ cells are critical for the improved abscopal effect seen by blocking/absence of $\beta 2$ adrenergic signaling. This critical role for $\mathrm{CD}^{+} \mathrm{T}$ cells also suggested that the effects we observed are tumor antigen-specific. To test this assumption, BALB/c mice cured by Prop and radiation (with both irradiated and distant tumors having disappeared for at least 2 months) were rechallenged with either the same tumor used earlier in these mice, CT26 or a different tumor, $4 \mathrm{~T} 1$. Figure $2 \mathrm{c}$ showed that compared to naïve mice, the tumor growth of local and distant CT26 tumor rechallenge was inhibited in all previously cured mice (Fig. 2c, left). When mice previously cured of CT26 tumor were subsequently rechallenged with $4 \mathrm{~T} 1$ cells, growth of 4T1 tumor was not inhibited (Fig. 2c, right), indicating that the growth inhibition was tumor-specific.

To investigate which immune cells are primarily responsible for the control of subsequent rechallenges, cured mice were treated with $\mathrm{CD}^{+}$or $\mathrm{CD}_{4}^{+}$depleting antibody prior to reinjection with CT26 tumor cells. We found that the cured mice depleted of $\mathrm{CD}^{+} \mathrm{T}$ cells, but not $\mathrm{CD} 4^{+} \mathrm{T}$ cells, did not reject either local or distant rechallenges (Fig. $2 \mathrm{~d}$ ), indicating that $\mathrm{CD}^{+}$ $\mathrm{T}$ cells are the key players for induction of memory responses against local and distant tumor rechallenges. As a result of these experiments, we determined that $\mathrm{CD} 8^{+} \mathrm{T}$ cells are crucial for the improved abscopal effect by blocking/absence of $\beta 2$-adrenergic signaling and also for inducing memory response in a tumorspecific manner.

Immune status following RT is dependent upon $\beta 2$-AR signaling. To explore the underlying cellular events by which reduced $\beta 2$-adrenergic signaling enhances anti-tumor immunity, we enumerated effector $\mathrm{CD}^{+} \mathrm{T}$ cells in tissues isolated from mice in the experiments presented above with flow cytometry. In the distant non-irradiated tumors from irradiated WT mice treated with Prop as well as irradiated $32-\mathrm{AR}$ KO mice treated with or without Prop, interferon gamma ${ }^{+}\left(\mathrm{IFN} \gamma^{+}\right)$, granzyme $\mathrm{B}^{+}$ $\left(\mathrm{GzmB}^{+}\right)$, tumor necrosis factor ${ }^{+}\left(\mathrm{TNFa}^{+}\right)$, and $\mathrm{T}$-box transcription factor ${ }^{+}\left(\mathrm{T}\right.$-bet $\left.{ }^{+}\right)$intra-tumoral effector $\mathrm{CD}^{+} \mathrm{T}$ cells significantly increased (Fig. 3a-d, Supplementary Fig. 2a), while M2 macrophages decreased (Supplementary Fig. 3). This suggests that blocking of $\beta 2$-adrenergic signaling enhances $\mathrm{T}$ cell-mediated anti-tumor immune responses in distant non-irradiated tumors while reducing the presence of M2 macrophages which are been shown to be immunosuppressive and pro-tumorigenic ${ }^{39-41}$. To assess the production of cytokines, which is an indicator of overall immune status, we measured the levels of IFN $\gamma$ and TNFa in the serum from the mice treated with $\beta$-blocker and radiation as well as the $\beta 2-\mathrm{AR} \mathrm{KO}$ mice treated with radiation. Figure $3 \mathrm{e}$ and $\mathrm{f}$ showed that both IFN $\gamma$ and TNFa levels increased in these mice. We also checked chemokine receptor $\mathrm{C}-\mathrm{X}-\mathrm{C}$ chemokine receptor type 3 (CXCR3) on $\mathrm{CD}^{+} \mathrm{T}$ cells in distant non-irradiated tumor and its ligand C-X-C motif chemokine 9 (CXCL9) in serum, which are associated with $\mathrm{CD} 8^{+} \mathrm{T}$ cell egress ${ }^{42-44}$, and found that both CXCR3 and CXCL9 increased in irradiated $\beta 2$-AR KO mice 


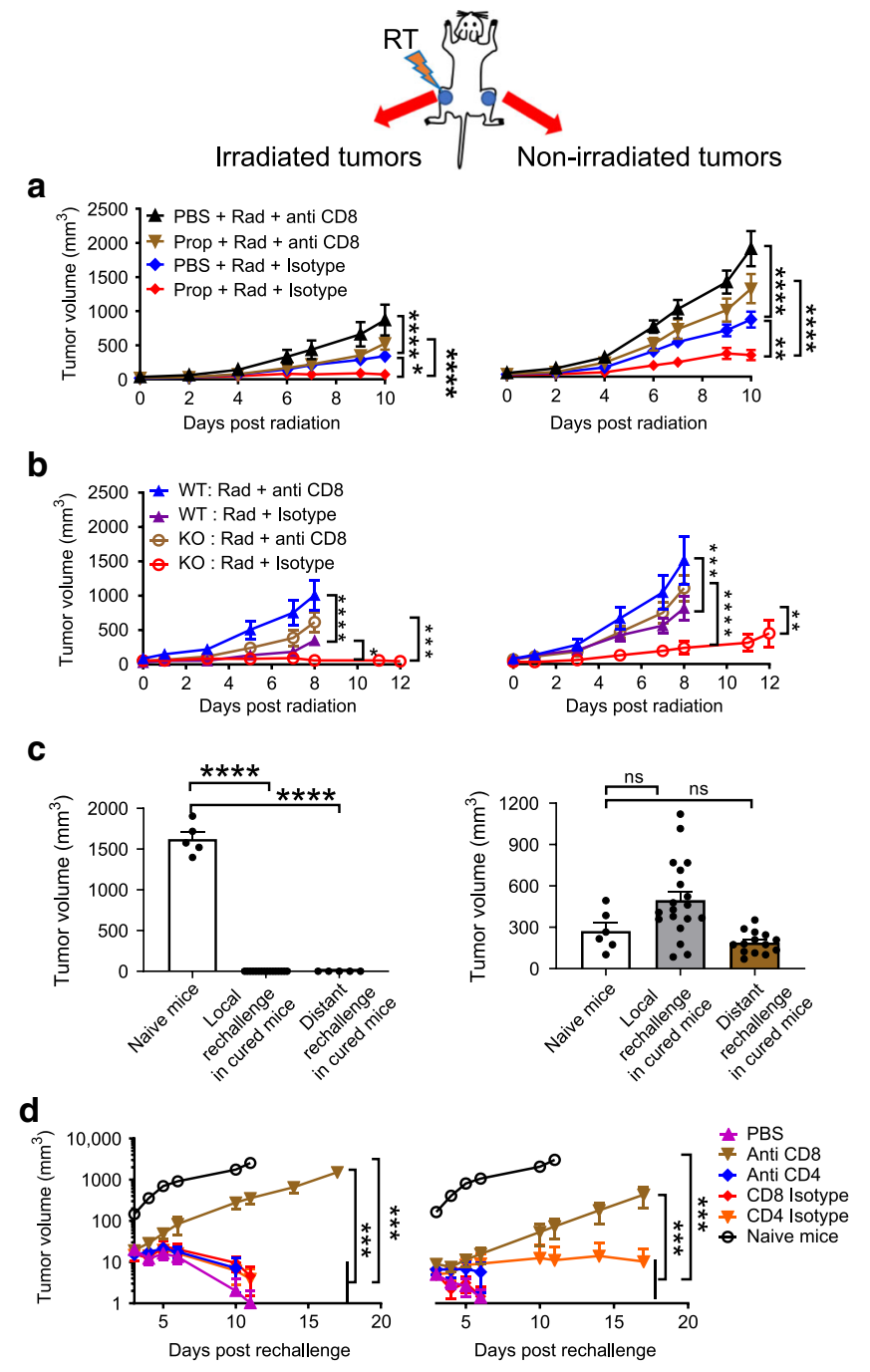

Fig. $2 \mathrm{CDB}^{+} \mathrm{T}$ cells are crucial for the improved abscopal effect achieved by blocking/absence of $\boldsymbol{\beta 2}$-adrenergic signaling in $\mathrm{CT} 26$ colon carcinoma model. a and $\mathbf{b}$ Growth of irradiated (left) and non-irradiated (right) tumors in irradiated WT mice treated with or without propranolol $\mathbf{a}$ and irradiated WT mice and $\beta 2-A R K O$ mice $\mathbf{b}$, which were depleted of $C D 8^{+}$ T cells. c The tumor growth of local and distant rechallenges with CT26 (left) and 4T1 (right) in mice cured by radiation plus propranolol compared with naïve mice. d The tumor growth (a log scale) of local (left) and distant (right) rechallenges with CT26 in mice cured by radiation and propranolol depleted of $\mathrm{CD}^{+}$or $\mathrm{CD} 4^{+} \mathrm{T}$ cells. Data are presented as mean \pm SEM. ${ }^{\star} P<0.05$; ${ }^{\star \star} P<0.01$; ${ }^{\star \star \star} P<0.001 ;{ }^{\star \star \star \star} P<$ $0.0001 ; n s=$ not significant (two-way ANOVA analysis for $\mathbf{a}, \mathbf{b}, \mathbf{d}$; oneway ANOVA analysis for $\mathbf{c}$ ). For $\mathbf{a}, n=10$ biologically independent mice in all groups; for $\mathbf{b}, n=9$ biologically independent mice in WT Rad + antiCD8 and WT Rad + isotype groups, $n=8$ biologically independent mice in KO Rad + anti-CD8 group, $n=10$ biologically independent mice in KO Rad + isotype group; for CT26 model in c, $n=5$ biologically independent mice in naïve mice group, $n=16$ biologically independent mice in local rechallenge in cured mice group, $n=5$ biologically independent mice in distant rechallenge in cured mice group; for $4 \mathrm{~T} 1$ model in $\mathbf{c}, n=6$ biologically independent mice in naïve mice group, $n=19$ biologically independent mice in local rechallenge in cured mice group, $n=14$ biologically independent mice in distant rechallenge in cured mice group; for $\mathbf{d}, n=6$ biologically independent mice in PBS, anti-CD8, anti-CD4, CD8 isotype, and CD4 isotype groups, $n=5$ biologically independent mice in naïve mice group. compared to irradiated WT mice (Fig. $3 \mathrm{~g}$ and $\mathrm{h}$ ), supporting the possibility that absence of $\beta 2$-AR signaling enhances the migration of $\mathrm{CD}^{+} \mathrm{T}$ cells by CXCR3/CXCL9 interactions.

To further explore the impact of adrenergic signaling on these and other molecules, we compared gene expression in CT26 tumors implanted bi-laterally in both WT mice and $\beta 2-\mathrm{AR} \mathrm{KO}$ mice, followed by radiation to just one of the tumors in each mouse. Shown in Fig. $3 \mathrm{i}$ and $\mathrm{j}$ is the $\log _{2}$ (fold change of the ratio of $\mathrm{WT} / \mathrm{KO}$ ) from $\mathrm{CD} 8^{+} \mathrm{T}$ cells. For the non-irradiated tumor in $\beta 2-\mathrm{AR} \mathrm{KO}$ mice (Fig. 3i), we noted a significant increase in expression of several genes including $C d 28$, Il2, and Zap70; genes showing the greatest decrease included genes which can inhibit effector function (including Il6 and Il10). For a complete list of differentially expressed genes, see Supplementary data 1. Importantly, IFN $\gamma$-related genes Cxcr3 increased in the non-irradiated tumors of $\beta 2$-AR KO mice, confirming the serum expression data above and supporting the idea that $\mathrm{T}$ cell migration from draining lymph node (LN) to tumors is enhanced in the absence or reduction of adrenergic signaling.

For irradiated tumors of $\beta 2-\mathrm{AR} \mathrm{KO}$ mice, expression of genes essential for effector function, including $C d 28, C d 86, I l 2, G z m b$, $C d 3 d$, and $\mathrm{T}$ cell migration, including Sele, Csf2, Cxcr6, Cd44, showed increased expression (Fig. $3 \mathrm{j}$ and Supplementary data 1). Cxcr3 3 and its ligand $\mathrm{Cxcl} 9$ were also seen to be increased in irradiated tumors of $\beta 2-\mathrm{AR} \mathrm{KO}$ mice. The significantly changed genes were used as input for Gene Ontology (GO) and pathway analysis. The upregulated genes in non-irradiated tumor of $\beta 2$ AR KO mice in the red module in Supplementary Fig. 4a were related to $\mathrm{T}$ cell receptor signaling pathway [false discovery rate $(\mathrm{FDR})=1.41 \times 10^{-4}$ ], receptor signaling pathway via Janus kinase-signal transducer and activator of transcription (JAKSTAT $) \quad\left(\mathrm{FDR}=1.70 \times 10^{-6}\right)$, positive regulation of tyrosine phosphorylation of STAT protein $\left(\mathrm{FDR}=1.62 \times 10^{-5}\right)$, positive regulation of nuclear factor kappa $B(N F \kappa B)$ transcription factor activity $\left(\mathrm{FDR}=5.91 \times 10^{-4}\right)$, positive regulation of interleukin 2 (IL-2) biosynthetic process $\left(\mathrm{FDR}=3.25 \times 10^{-6}\right)$, positive regulation of activated $\mathrm{T}$ cell proliferation $\left(\mathrm{FDR}=3.76 \times 10^{-5}\right)$. On the other hand, the red module in Supplementary Fig. $4 \mathrm{~b}$ also showed that the upregulated genes in irradiated tumor of $\beta 2$-AR KO mice were related to similar important regulatory functions.

Role of tumor draining LNs in enhanced abscopal effects. A current proposed mechanism by which radiation can increase anti-tumor immunity suggests that damage to the tumor from ionizing radiation increases antigen availability and uptake by dendritic cells which then travel to draining LNs where they activate naïve, antigen-specific $\mathrm{T}$ cells. Effector $\mathrm{T}$ cells must then re-enter the circulation from the draining LNs to target antigenexpressing tumor cells ${ }^{38,45}$. However, recent studies show that $\beta 2$ AR adrenergic signaling in activated $T$ cells inhibits their exit from LNs and suggests that this occurs by enhancement of retention promoting signals ${ }^{46,47}$. Thus, to investigate the role of $\mathrm{T}$ cell egress from draining LNs in the mechanism underlying enhanced abscopal effects achieved by combining radiation with reduced $\beta$-AR signaling, we used both WT mice (at TT) or $\beta 2$-AR $\mathrm{KO}$ mice (at ST) as models of reduced AR signaling. Mice with bilateral tumors received RT to one tumor and were treated with FTY720, a sphingosine 1-phosphate receptor (S1PR) agonist which is phosphorylated by cellular sphingosine kinases and binds to S1PR resulting in receptor internalization and degradation. This drug is well recognized to prevent $\mathrm{T}$ cell egress from lymphoid tissues leading to decreased numbers of lymphocytes in non-lymphoid peripheral tissues ${ }^{48-50}$. We observed that FTY720 
a

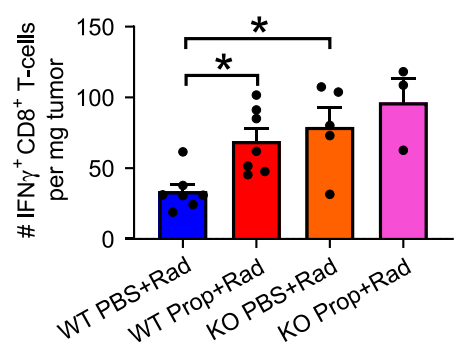

C

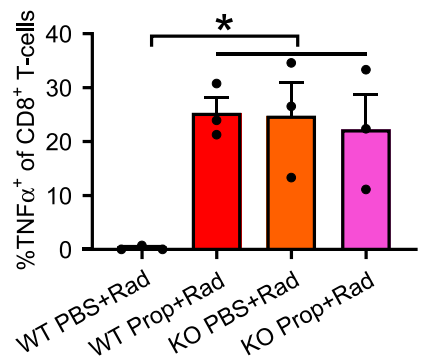

b

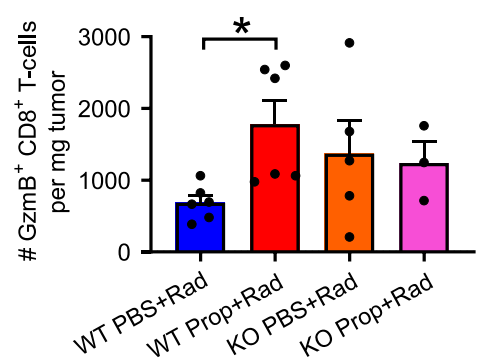

d

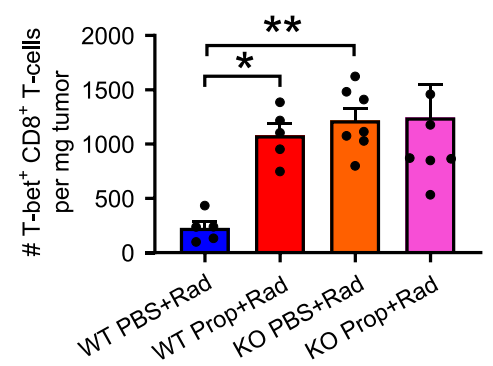

$\mathbf{e}$

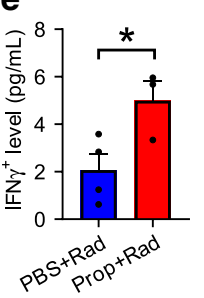

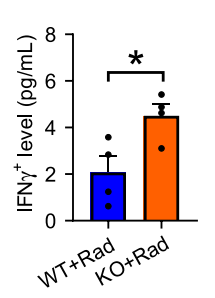
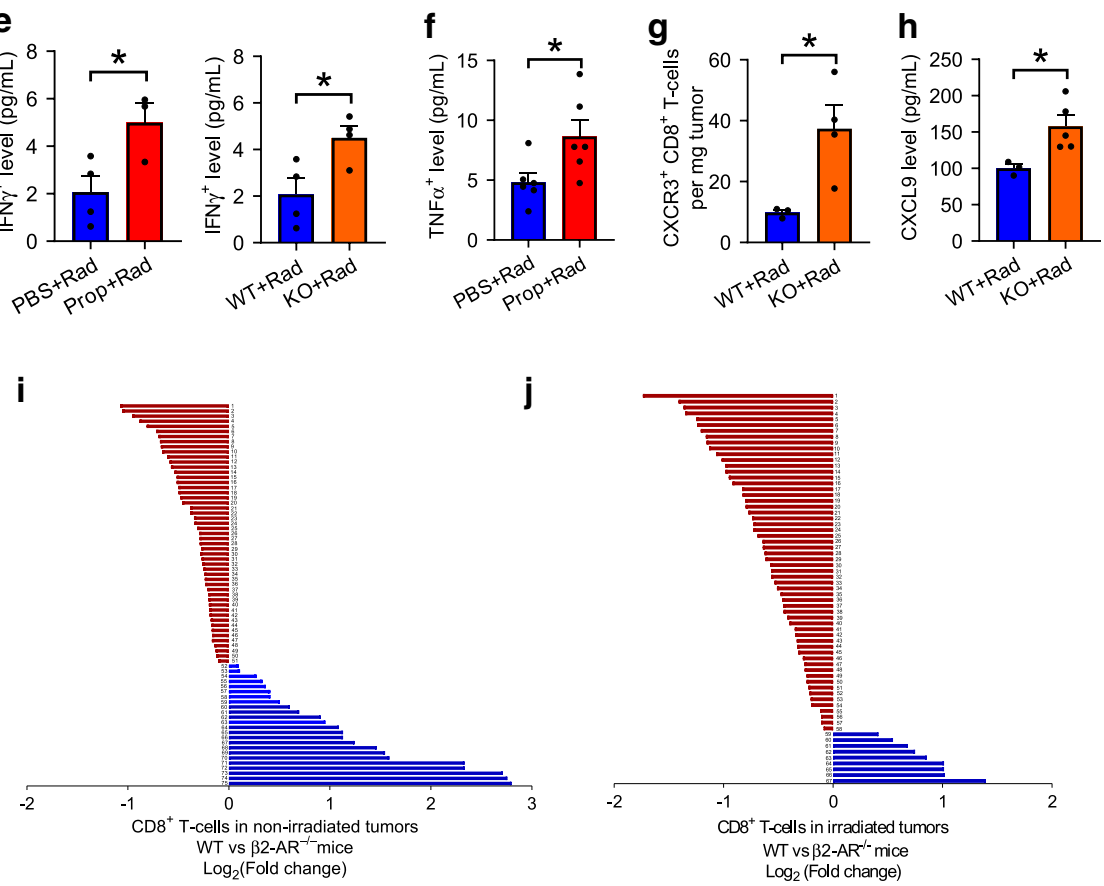

Fig. 3 Blocking/absence of $\beta 2$-adrenergic signaling increases expression of genes involved in enhancing the cell-mediated anti-tumor immune response and T cell egress in non-irradiated CT26 tumors. Immune analyses were performed in non-irradiated tumors of irradiated WT or $\beta 2$-AR KO mice treated with or without propranolol. Reduction or absence of $\beta 2$-adrenegic signaling increased the expression of important effector molecules in CD8 ${ }^{+}$T cells including: a IFN $\gamma^{+}, \mathbf{b} \mathrm{GzmB}^{+}, \mathbf{c} \mathrm{TNF} \alpha^{+}$, and $\mathbf{d}$ T-bet ${ }^{+}$. The level of IFN- $\gamma$ was detected in serum of irradiated WT mice treated with or without propranolol (e, left panel) or irradiated WT and $\beta 2-A R$ KO mice (e, right panel) as well as TNF $\alpha$ in irradiated mice treated with or without propranolol f. The expression of CXCR3 in non-irradiated tumors $\mathbf{g}$ and the level of CXCL9 in serum $\mathbf{h}$ was detected in irradiated WT or $\beta 2$-AR KO mice. $\mathbf{i}$ and $\mathbf{j}$ Gene profiles with NanoString in CD8 ${ }^{+}$T cells (data are presented as $\log _{2}$ (fold change of WT/KO)) of the non-irradiated tumors $\mathbf{i}$ and irradiated tumors $\mathbf{j}$ from WT and $\beta 2$-AR KO mice which had been given radiation to the contralateral tumors. Detailed information is shown in Supplementary data 1. Data are presented as mean \pm SEM. ${ }^{\star} P<0.05 ;{ }^{\star}{ }^{*} P<0.01$ (one-way ANOVA analysis for $\mathbf{a}-\mathbf{d}$; Student's $t$ test analysis for $\mathbf{e}-\mathbf{h}$ ). For $\mathbf{a}, n=7$ biologically independent mice in WT PBS + Rad and WT Prop + Rad groups, $n=5$ in KO PBS + Rad group, $n=3$ in KO Prop + Rad group; for $\mathbf{b}, n=6$ biologically independent mice in WT PBS + Rad and WT Prop + Rad groups, $n=5$ in KO PBS + Rad group, $n=3$ in KO Prop + Rad group; for $\mathbf{c}, n=3$ biologically independent mice in all groups; for d, $n=5$ biologically independent mice in WT PBS + Rad and WT Prop + Rad groups, $n=7$ in KO PBS + Rad and KO Prop + Rad groups; for e, $n=4$ biologically independent mice in PBS + Rad, WT + Rad, and KO + Rad groups, $n=3$ in Prop + Rad group; for $\mathbf{f}, n=6$ biologically independent mice in all groups; for $\mathbf{g}, n=3$ biologically independent mice in WT + Rad group, $n=4$ in KO + Rad group; for $\mathbf{h}, n=3$ biologically independent mice in WT + Rad group, $n=5$ in $\mathrm{KO}+$ Rad group; for $\mathbf{i}$ and $\mathbf{j}, n=8$ biologically independent mice in WT + Rad and KO + Rad groups. 


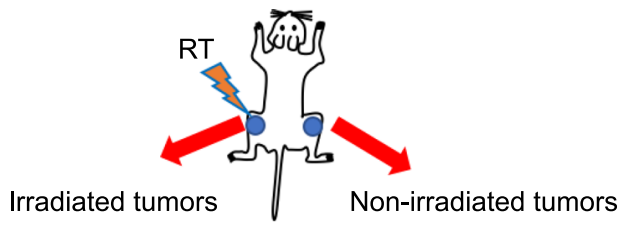

a

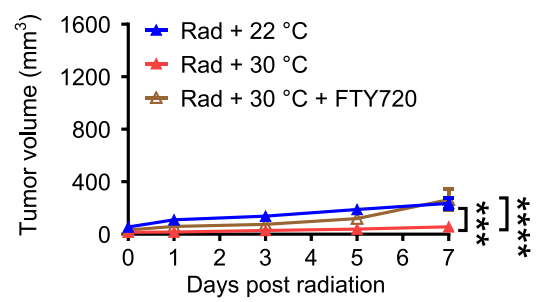

b

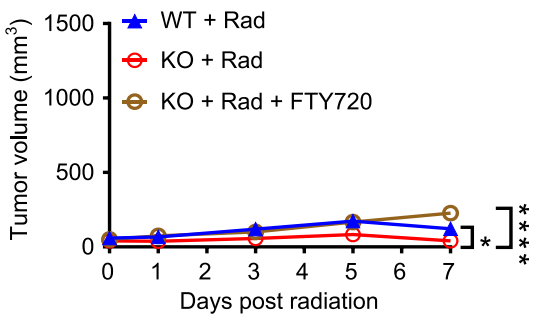

C
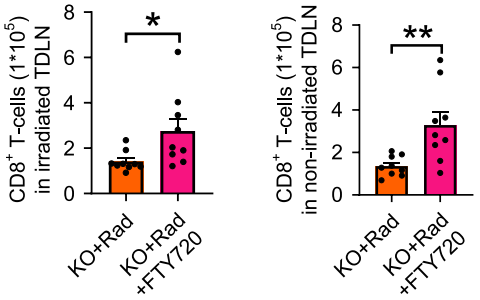

e
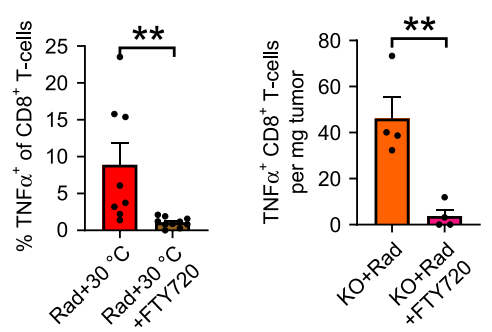

g
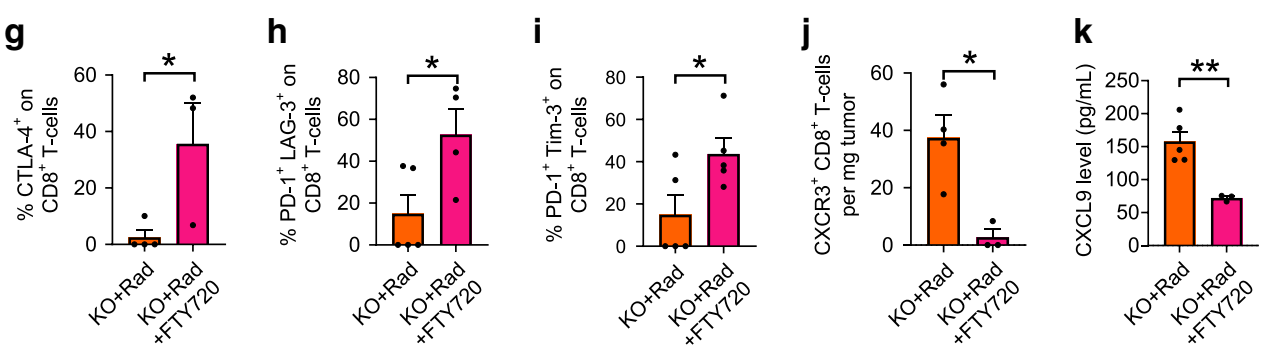

treatment resulted in a significant loss of the enhanced tumor control seen by housing mice at $30^{\circ} \mathrm{C}$ (CT26, Fig. 4a; B16, Supplementary Fig. 5a, b). Similarly, the enhanced immune control of tumors induced by RT in $\beta 2$-AR KO mice was also lost in mice treated with FTY720 (Fig. 4b). Analysis of $\mathrm{CD} 8^{+} \mathrm{T}$ cells by flow cytometry confirmed increased numbers of $\mathrm{CD}^{+} \mathrm{T}$ cells retained in the draining LNs of both irradiated and distant non-irradiated tumors in KO mice treated with FTY720 (Fig. 4c). These results support a role for T cells which had been in the draining LN (and not simply present in the nearby tissues or blood) in generating

improved systemic immunity following radiation in the absence of adrenergic stress signaling.

To further our understanding of how FTY720 and adrenergic signaling influences the immune landscape in tumors, we characterized expression of various effector molecules and coinhibitory receptors on Day 7 post-RT using flow cytometry in mice treated or non-treated with FTY720. As shown in Fig. 4d-f (left panel), FTY720 treatment was associated with fewer IFN $\gamma^{+}$, $\mathrm{TNF}^{+}$, and $\mathrm{GzmB}^{+} \mathrm{CD}^{+}{ }^{+} \mathrm{T}$ cells in these tumors compared with those of control mice housed at $30^{\circ} \mathrm{C}$. Similar results were obtained 
Fig. 4 32-adrenergic signaling modulates the abscopal effect via the egress of CD8 ${ }^{+} \mathbf{T}$ cells from the $\mathbf{L N}$ in the CT26 colon carcinoma model. Growth of irradiated tumors (left) and non-irradiated tumors (right) in the mice housed at $22^{\circ} \mathrm{C}$ (ST) or $30^{\circ} \mathrm{C}$ (TT) that did or did not receive FTY720 a; or in WT or $\beta 2-A R K O$ mice which were irradiated and then did or did not receive FTY720 b; c CD8 ${ }^{+}$T cells in the draining $L N$ of irradiated (left) and non-irradiated (right) tumors in irradiated $\beta 2-A R$ KO mice treated with/without FTY720; d-f Data from irradiated WT mice housed at $30^{\circ} \mathrm{C}$ (left panels) and $\beta 2-A R$ KO mice (right panels) which received radiation with/without FTY720: expression of IFN $\gamma^{+} \mathbf{d}$, TNF $\alpha^{+} \mathbf{e}$, and $\mathrm{GzmB}{ }^{+} \mathbf{f} ; \mathbf{g}^{-i}$ the expression of CTLA-4 ${ }^{+} \mathbf{g}$, PD-1 ${ }^{+}$ and LAG- $3^{+} \mathbf{h}, \mathrm{PD}-1^{+}$, and Tim- $3^{+} \mathbf{i}$ in non-irradiated tumors from irradiated $\beta 2-\mathrm{AR}$ KO mice treated with/without FTY720. The expression of CXCR3 in non-irradiated tumors $\mathbf{j}$ and the level of CXCL9 in serum $\mathbf{k}$ was detected in irradiated $\beta 2-A R$ KO mice treated with/without FTY720. Data are presented as mean \pm SEM. ${ }^{\star} P<0.05 ;{ }^{\star \star} P<0.01$; ${ }^{\star \star \star} P<0.001 ;{ }^{\star \star \star \star} P<0.0001$ (two-way ANOVA analysis for $\mathbf{a}, \mathbf{b}$; Student's $t$ test analysis for $\mathbf{c}-\mathbf{k}$ ). For $\mathbf{a}, n=10$ biologically independent mice in all groups; For $\mathbf{b}$ and $\mathbf{c}, n=9$ biologically independent mice in all groups; For $\mathbf{d}$ and $\mathbf{f}, n=8$ biologically independent mice in $\operatorname{Rad}+30^{\circ} \mathrm{C}$ group, $n=10$ biologically independent mice in Rad $+30^{\circ} \mathrm{C}+\mathrm{FTY} 720$ group, $n=3$ biologically independent mice in KO Rad group, $n=4$ biologically independent mice in KO + Rad + FTY720 group; For e, $n=8$ biologically independent mice in Rad $+30^{\circ} \mathrm{C}$ group, $n=10$ biologically independent mice in Rad $+30^{\circ} \mathrm{C}+\mathrm{FTY} 720$ group, $n=4$ biologically independent mice in $\mathrm{KO}+$ Rad group, $n=4$ biologically independent mice in $\mathrm{KO}+$ Rad + FTY720 group; For $\mathbf{g}$ and $\mathbf{j}, n=4$ biologically independent mice in KO + Rad group, $n=3$ biologically independent mice in KO + Rad + FTY720 group; For $\mathbf{h}, n=5$ biologically independent mice in KO + Rad group, $n=4$ biologically independent mice in KO + Rad + FTY720 group; For $\mathbf{i}, n=5$ biologically independent mice in two groups; For $\mathbf{k}, n=5$ biologically independent mice in $\mathrm{KO}+$ Rad group, $n=3$ biologically independent mice in $\mathrm{KO}+$ Rad + FTY720 group.

from irradiated $\beta 2-\mathrm{AR} \mathrm{KO}$ mice (Fig. $4 \mathrm{~d}-\mathrm{f}$ right panel), however, we also found that expression of co-inhibitory molecules (cytotoxic T-lymphocyte-associated protein 4 (CTLA-4), programmed cell death-1 (PD-1), lymphocyte-activation gene 3 (LAG-3) and T cell immunoglobulin and mucin-domain containing-3 (Tim-3)) was significantly increased (Fig. $4 \mathrm{~g}-\mathrm{i}$ ). Together, these data support the possibility that adrenergic signaling sequesters effector $\mathrm{T}$ cells in the draining $\mathrm{LN}^{46,47}$, and reversal of suppression allows migration of these $\mathrm{T}$ cells into the tumor thereby allowing development of an abscopal response. Moreover, as shown in Fig. $4 \mathrm{j}$ and $\mathrm{k}$ and Supplementary Fig. 2b, CXCR3 ${ }^{+} \mathrm{CD} 8^{+} \mathrm{T}$ cells (in the nonirradiated tumor) and CXCL9 (in the serum) were decreased in FTY720 treated, irradiated $\beta 2$-AR KO mice compared to controls, suggesting that CXCR3/CXCL9 may play an important role in $\mathrm{CD}^{+} \mathrm{T}$ cell migration to tumors in the absence of $\beta 2$-AR expression. Similar results were also observed in B16 model (Supplementary Fig. 6a-d). Taken together, these data lead to the speculation that one mechanism by which $\beta 2$-adrenergic signaling suppresses the abscopal effect is by retention of $\mathrm{CD}^{+} \mathrm{T}$ cells within the tumor draining LNs, as has been seen in the experimental autoimmune encephalomyelitis model of autoimmune disease ${ }^{46}$. However, this potential mechanism is based on correlative data so far, and further study is required to establish a causative role for adrenergic stress in specific patterns of immune cell migration or trafficking to the tumor microenvironment.

$\beta$-blocker improves combination of RT and checkpoint blockade. After determining that $\beta$-blocker enhances abscopal effect and is associated with decreased PD- 1 expression on CD8 ${ }^{+}$ $\mathrm{T}$ cells, we then investigated whether $\beta$-blocker can further improve radiation responses, especially abscopal effect, in antiPD-1 checkpoint blockade immunotherapy. The mice were implanted with CT26 tumor cells and treated with Prop or PBS as described before. After local radiation of one tumor, the mice were treated with six doses of anti-PD-1 or isotype control antibodies. A detailed experimental design is shown in Supplementary Fig. 7a. Comparison of tumor growth (Supplementary Fig. 7b) showed that compared to PBS, Prop significantly enhanced the tumor control of irradiated and non-irradiated tumors in the mice treated with anti-PD-1 antibody, suggesting that adrenergic stress interferes with the effects of the combination of radiotherapy and immunotherapy, which can be overcome by the treatment of $\beta$-blocker.

$\beta$-blocker reduces metastatic spread after local RT. Thus far, we have used implantable tumor models to investigate the impact of $\beta$-AR signaling on control by radiation of both irradiated and non-irradiated, distant tumors. Naturally occurring metastatic tumors offer an important opportunity to study factors which affect the abscopal effect. Thus, to investigate whether $\beta$-AR blockade affects metastases after radiation to the primary tumor, we employed the 4T1-Luc model to repeat the experiment with the treatment of Prop and radiation. 4T1 tumors exhibit metastatic spread to the lungs following their orthotopic implantation to the mammary glands of mice ${ }^{32,51}$. As shown in Fig. $5 \mathrm{a}$, compared with PBS group, $\beta$-blocker Prop treatment significantly improved the tumor control of both irradiated and non-irradiated mammary tumors after $12 \mathrm{~Gy}$ local radiation to one of the orthotopically implanted tumors. On Day 33 post tumor implantation, bioluminescence imaging (BLI) of luciferase activity (Fig. 5b) showed that Prop combined with radiation given earlier to an orthotopically implanted mammary tumor also reduced lung metastases. Moreover, analysis of lung tissue morphology on haemotoxylin and eosin ( $\mathrm{H} \& \mathrm{E})$-stained histologic sections also indicated fewer metastatic lesions in the Prop-treated group (Fig. 5c). These data suggest that blockade of $\beta$-AR signaling reduces spontaneous metastases after radiation to the primary tumor.

\section{Discussion}

RT used as a single agent is now thought to promote immunity against tumors, including increased generation of antigen-specific effector T cells. However, a common belief is that radiation alone is not sufficient to induce a therapeutically effective, systemic $\mathrm{T}$ cell response capable of controlling distant, non-irradiated tumors (i.e., an abscopal effect). The data presented here provide a counter-argument to this widespread assumption.

Three conclusions can be drawn from the data presented here. First, the baseline adrenergic stress caused by cool housing temperature mandated for laboratory mice for decades is immunosuppressive and has led to a significant underestimation of the natural ability of radiation alone to elicit an abscopal effect. This point alone should alert the radiation biology community to the dependence of experimental outcomes on physiological and environmental factors which are largely ignored by researchers. Using a combination of pharmacological and genetic approaches, our second finding is that the mechanism by which cool housing temperature influences the abscopal effect is via AR signaling leading to immunosuppression. This includes a demonstration of enhanced control of metastatic tumors in the $4 \mathrm{~T} 1$ mammary tumor model where radiation was delivered to the primary tumor in the presence of Prop. Third, the enhancement of the abscopal effect achieved by reducing adrenergic signaling is dependent upon $\mathrm{CD}^{+} \mathrm{T}$ cells, and perhaps the response of other immune 
a

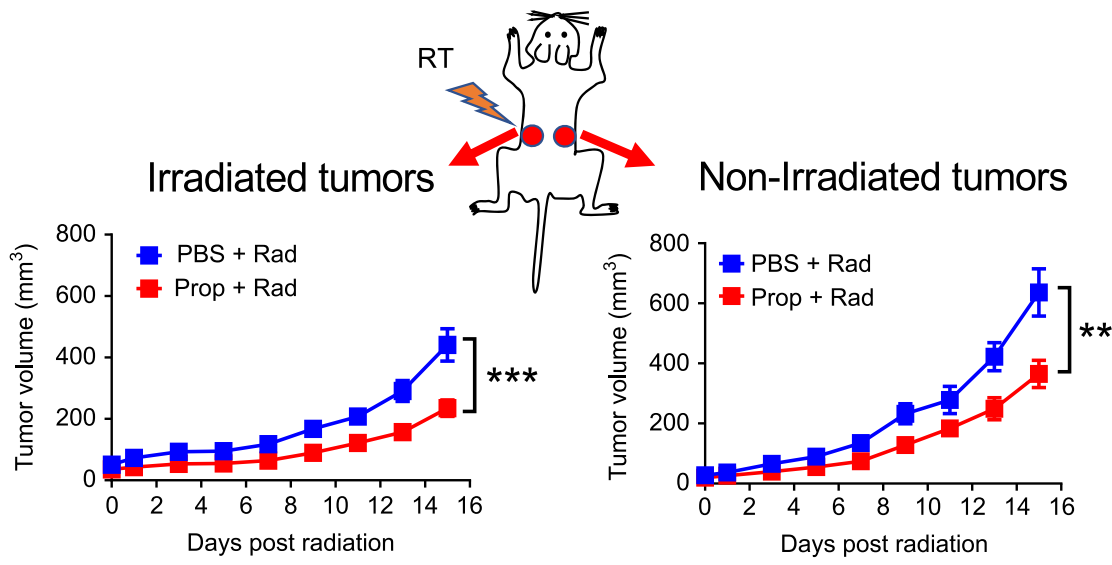

b

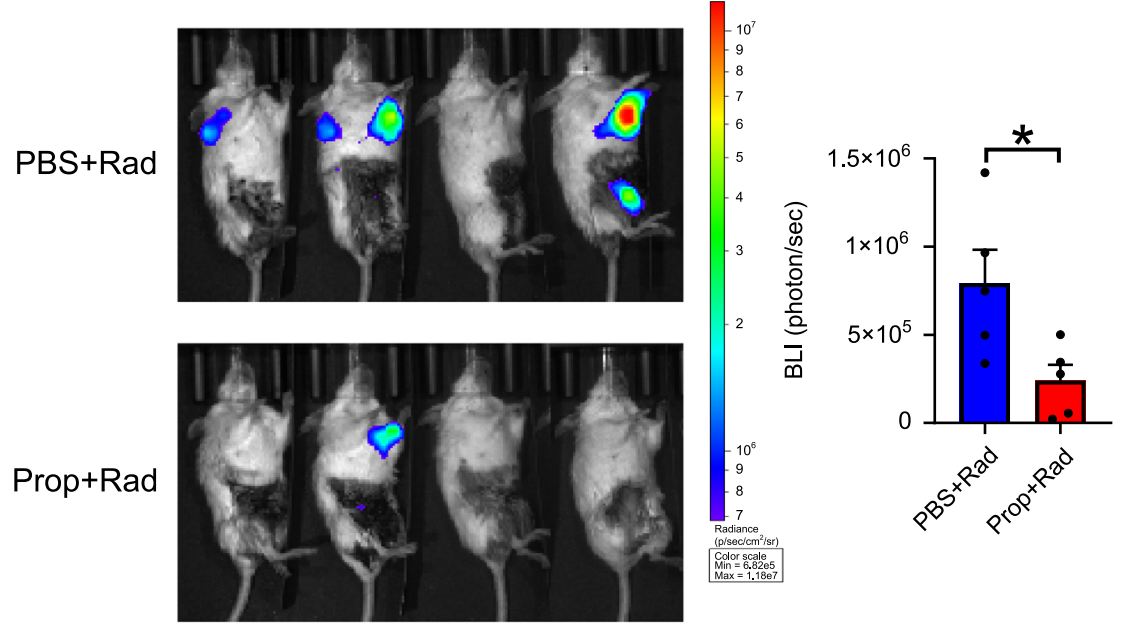

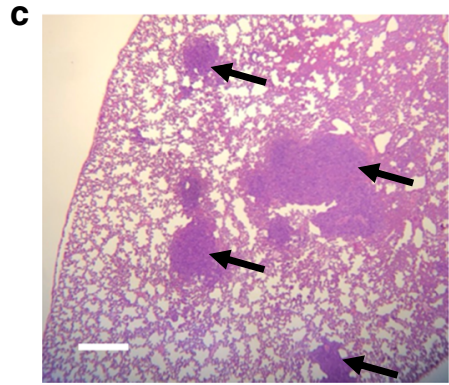

PBS+Rad

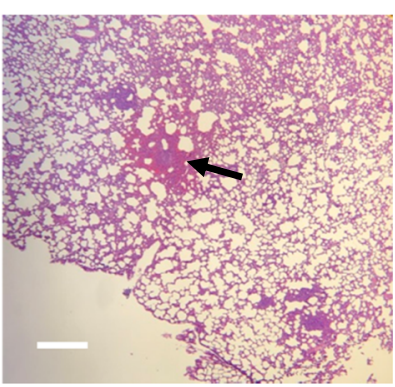

Prop+Rad

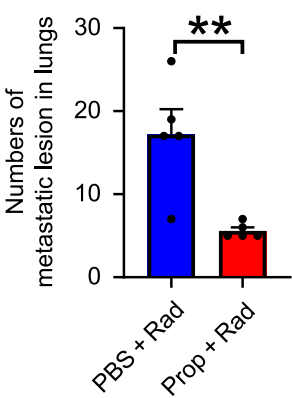

Fig. 5 Blockade of $\boldsymbol{\beta}$-adrenergic signaling reduces spontaneous metastases in the 4T1-luc breast carcinoma model. a Tumor growth of irradiated tumors (left) and non-irradiated tumors (right) is shown. Data are presented as mean \pm SEM. ${ }^{\star \star} P<0.01 ;{ }^{\star \star \star} P<0.001$ (two-way ANOVA analysis). $n=12$ biologically independent mice in PBS + Rad group and $n=18$ biologically independent mice in Prop + Rad group. On Day 33 post tumor implantation, bioluminescent images of luciferase activity $\mathbf{b}$ were acquired and histologic analysis of lung tissues was shown in $\mathbf{c}$. Staining with eosin hematoxylin was performed ( $\times 40$ magnification, scale bars $=500 \mu \mathrm{m}$ ) and black arrows showed metastatic lesions. Data are presented as mean \pm SEM. ${ }^{\star} P<0.05 ;{ }^{\star \star} P<$ 0.01 (Student's $t$ test analysis). For $\mathbf{b}$ and $\mathbf{c}, n=5$ biologically independent mice in all groups.

cell types, and is associated with significant beneficial changes in the immune status of irradiated and non-irradiated tumors. This results in an immune system that is more capable of controlling tumors outside the field of radiation. Together, these data reveal an unexpected degree of control by the sympathetic nervous system over the efficacy of radiation, both in irradiated tumors and those at distant non-irradiated sites. Thus, adrenergic stress signaling in the tumor may be included with other known physiological factors that influence the efficacy of RT, such as intratumoral hypoxia or metabolic activity ${ }^{52-54}$. These data also provide additional evidence for the importance of tumor immunity in mediating the overall outcome of RT and abscopal events.

The precise mechanisms by which blockade or absence of $\beta-A R$ signaling enhances systemic anti-tumor immunity following local radiation remain to be established. Data presented here show a critical role for $\mathrm{CD} 8{ }^{+} \mathrm{T}$ cells with upregulation of T-bet transcription, increased secretion of IFN $\gamma, \mathrm{TNF} \alpha$ and GzmB and upregulation of CXCR3/CXCL9 signaling, which is required for effector $\mathrm{T}$ cell migration across tumor vessels ${ }^{55}$. This adds to our previously published data (in non-radiation settings) that blockade of $\beta$-AR signaling increases metabolic activity during $\mathrm{CD} 8^{+} \mathrm{T}$ 
cell activation ${ }^{33}$. Further, we have observed that the addition of Prop reduces the frequency of immunosuppressive M2 macrophages in the non-irradiated tumor following radiation. Macrophages have previously been shown to be regulated by adrenergic stress $^{56,57}$ and M2 macrophages in particular have been linked to enhanced immunosuppression ${ }^{39-41}$. This is consistent with our other data that the blockade of adrenergic stress suppresses the recruitment, survival, and function of myeloid-derived suppressor cells (MDSCs) and reduces the frequency of intratumoral regulatory T cells (Tregs) $29,32,58$. Thus, the combination of enhanced activity of $\mathrm{CD}^{+}{ }^{+} \mathrm{T}$ cells combined with reduced immunosuppression in mice resulting from reduced $\beta$-AR signaling could result in a major tilt of the immune balance toward systemic tumor eradication following local radiation.

Preclinical models of many tumor types have shown that radiation can increase recognition of malignant cells through a variety of mechanisms including enhanced antigen uptake and presentation by dendritic cells ${ }^{59,60}$. Our data show that the enhanced abscopal effect following reduction or blockade of adrenergic signaling is dependent on $\mathrm{T}$ cell egress from tumor draining LNs. This supports a model ${ }^{38,45}$ in which radiation stimulates antigen-dependent activation of naïve $\mathrm{T}$ cells in draining LNs, which then must exit the LNs and migrate to the tumor microenvironment. However, these data are still indirect and correlative. Experiments which directly demonstrate a causal regulation of $\mathrm{T}$ cell, migration patterns and trafficking to the tumor microenvironment by adrenergic stress are still needed.

Other questions arise from these data. For example, what is the impact of adrenergic stress on the efficacy of different radiation schedules (e.g., fractionated vs. hypofractionated) and on antitumor immunity ${ }^{61}$ ? Here, we used single fraction doses that are known to be suboptimal in controlling tumor growth within the field of irradiation and do not result in abscopal effects. This is an important question to answer because in the clinic, some patients receive a single fraction of radiation, while others receive multiple fractions, and it is not clear how these protocols influence the development of anti-tumor immunity. Recently, single fraction RT (compared to surgery alone) for renal clear cell carcinoma patients demonstrated increased tumor associated antigen expression with a concomitant rise in the intratumoral frequency of proliferating $\mathrm{CD}^{+} \mathrm{T}$ cells ${ }^{62}$. These parameters will be important to examine in terms of the impact of adrenergic stress.

While our work here reveals that adrenergic stress is a major factor dictating the overall response to radiation in vivo, we have also observed that increased $\beta$-AR signaling through treatment with the agonist, isoproterenol, can directly increase tumor cell resistance to radiation in vitro ${ }^{37}$. Thus, how stress affects both direct (tumor cell intrinsic) as well as indirect targets (including anti-tumor immunity as demonstrated here), to regulate the overall radiation response in vivo remains to be established.

Recent studies have demonstrated that an abscopal effect can be induced in patients by the combination of radiotherapy and anti-PD1-therapy (nivolumab ${ }^{63,64}$, pembrolizumab ${ }^{65,66}$ ), antiCTLA-4 therapy (Ipilimumab ${ }^{67}$ ) and granulocyte-macrophage colony-stimulating factor (GM-CSF) therapy ${ }^{68}$. We previously found ${ }^{29}$ that combining Prop with anti-PD-1 in murine tumor models results in a significant improvement in anti-PD-1 efficacy. Thus, here we tested the triple combination of Prop with radiation and anti-PD-1. As we predicted, we found a significantly improved control of tumor growth by radiation and anti-PD-1 when Prop was included. Thus, future work must delineate precise interactions between radiation, immunotherapy delivery, and adrenergic stress in order to maximize therapeutic application of these combination therapies as soon as possible.

In summary, the immunosuppressive impact of cool housing temperature has masked the baseline ability of radiation alone to control tumor growth, especially tumors outside the field of radiation, in essentially all pre-clinical mouse models. However, the study of this phenomenon has led us to recognize the profound role of nerves, stress, and AR signaling in regulating the impact of radiation and provides a useful model with which to explore the impact of the immune system and chronic stress on the efficacy of RT. The data presented here also support the possibility that patients experiencing increased adrenergic stress (elicited by many stressors including anxiety, fear, and depression) following a cancer diagnosis may also be at risk for impaired responses to RT. Related to this possibility, previous retrospective, epidemiological studies show that co-incident use of $\beta$-blockers (primarily for cardiovascular conditions) was associated with decreased distant metastases and improved distant metastasis-free survival, disease-free survival, and overall survival in patients with non-small-cell lung cancer after radiotherapy ${ }^{69,70}$, while a recent retrospective study ${ }^{58}$ demonstrates that prior use of non-specific $\beta$-blockers enhances survival of patients receiving immunotherapy. These positive results could be linked to enhancement of immunological pathways by stress reduction shown here and strongly support implementation of prospective clinical trials combining $\beta$-AR blockade with radiation and/or immunotherapy. Prop is an inexpensive, widely used, and well tolerated drug. Consequently, there are few barriers to implementing many of the studies proposed above in the radiation oncology clinic.

\section{Methods}

Mice. 6-8 weeks old Female BALB/cand C57BL/6 were purchased from Charles River and SCID mice from the Laboratory Animal Resource at Roswell Park Comprehensive Cancer Center. BALB/c mice globally deficient in $\beta 2$-ARs $\left(A d r b 2^{-l-}\right)$ were provided by David Farrar (University of Texas Southwestern Medical Center). Mice were maintained in specific pathogen-free facilities and all studies were performed in accordance with all relevant ethical regulations for animal testing and research as well as the protocols approved by the IACUC at Roswell Park Comprehensive Cancer Center.

Cell culture and tumor implantation. CT26.CL25 tumor cells (CRL-2639 $9^{\text {rm }}$ ), B16 F10 tumor cells (CRL-6475 $5^{\mathrm{mm}}$ ), and 4T1 tumor cells (CRL-2539 $9^{\mathrm{mm}}$ ) were purchased from and authenticated by American Type Culture Collection (ATCC). 4T1-luc tumor cells were stably tagged with the luciferase gene by Genomics Shared Resource in Roswell Park Comprehensive Cancer Center. CT26.CL25 cells were cultured with Roswell Park Memorial Institute (RPMI) 1640 medium with $2 \mathrm{mM} \mathrm{L}$ glutamine adjusted to contain $1.5 \mathrm{~g} / \mathrm{L}$ sodium bicarbonate, $4.5 \mathrm{~g} / \mathrm{L}$ glucose, $10 \mathrm{mM}$ HEPES, $1.0 \mathrm{mM}$ sodium pyruvate, $0.1 \mathrm{mM}$ non-essential amino-acids, $0.4 \mathrm{mg} / \mathrm{mL}$ G418, and 10\% fetal bovine serum. B16-F10, 4T1-luc, and 4T1 cells were cultured in RPMI 1640 (Gibco) supplemented with 10\% FBS, 1\% L-glutamine, and 1\% penicillin/streptomycin. Once thawed, cells were cultured in an incubator at $37^{\circ} \mathrm{C}$ with $5 \%$ carbon dioxide and $95 \%$ air and passed twice prior to tumor implantation. $5 \times 10^{5}$ CT26.CL25 cells or $1 \times 10^{5}$ B16-F10 cells in $70 \mu \mathrm{L}$ PBS were subcutaneously injected into both hindlimbs of the mice. For rechallenge experiments, $5 \times 10^{5}$ CT26.CL25 cells or $1 \times 10^{4} 4 \mathrm{~T} 1$ cells were subcutaneously injected into both the hindlimb (in $70 \mu \mathrm{L}$ PBS, local rechallenge) and in the flank (in $100 \mu \mathrm{L}$ PBS, distant rechallenge) of the mice cured by the treatment of Prop and radiation for at least 2 months. For lung metastases experiment, $1 \times 10^{5} 4$ T1-luc cells were subcutaneously injected into bilateral 4 th mammary fat pad. Tumor growth was monitored by the measurement of perpendicular diameters (width/length) every 2 days and tumor volume was calculated with the formula ((width ${ }^{2} \times$ length)/2).

Irradiation of tumors. When tumors became palpable $\left(\sim 100 \mathrm{~mm}^{3}\right)$, one of the tumors per mouse received 6 Gy (CT26), 20 Gy (B16), or 12 Gy (4T1-luc) of 200 $\mathrm{kVp}$ x-ray local RT, using a Philips RT-250. The mice were placed in a lead shield to expose one of the tumors and prevent radiation from reaching any other part of the mouse.

Housing mice at different ambient temperatures. Mice were acclimated to either standard temperature $\left(\sim 22^{\circ} \mathrm{C}\right)$ or thermoneutral temperature $\left(\sim 30^{\circ} \mathrm{C}\right)$ for at least 3 weeks before tumor implantation in precision refrigerated plant-growth incubators (Thermo Fisher Scientific), in which humidity was maintained with a Top Fin ${ }^{\circledR}$ Air Pump AIR 1000 and Top Fin ${ }^{\circledR}$ tubing.

Treatment of Prop. Mice were intraperitoneally injected daily with $200 \mu \mathrm{g}$ Prop (P0884, Sigma-Aldrich) in $100 \mu \mathrm{L}$ of PBS while control mice received $100 \mu \mathrm{L}$ PBS daily from 3 days prior to irradiation until the endpoint of the experiments. 
Treatment of FTY720. Mice were treated by intragastric administration with $25 \mu \mathrm{g}$ FTY720 (BML-SL233-0005, Enzo) in $200 \mu \mathrm{L}$ of PBS while control mice received $200 \mu \mathrm{L}$ PBS 2 days prior to irradiation and then treated daily with $5 \mu \mathrm{g}$ FTY720 in $100 \mu \mathrm{L}$ of PBS while control mice received $100 \mu \mathrm{L}$ PBS daily until the endpoint of the experiments.

CD8 $^{+}$and CD4 ${ }^{+} \mathbf{T}$ cell depletion. Mice were treated weekly with $400 \mu \mathrm{g} I n V i-$ voMAb anti-mouse CD8a (53-6.72, BioXCell) or InVivoMAb anti-mouse CD4 (GK1.5, BioXCell) as experimental groups or with InVivoMAb rat IgG2a isotype control (2A3, BioXCell) or InVivoMAb rat IgG2b isotype control (LT F-2, BioXCell) as control groups by i.p. injections 4 days prior to tumor implantation. $\mathrm{CD}^{+}$ or $\mathrm{CD} 4^{+} \mathrm{T}$ cell depletion was confirmed by flow cytometry.

Combination immunotherapy experiment. $5 \times 10^{5}$ CT26.CL25 tumor cells were implanted as above-mentioned. Mice were intraperitoneally injected daily with $200 \mu$ g Prop (P0884, Sigma-Aldrich) in $100 \mu \mathrm{L}$ of PBS while control mice received $100 \mu \mathrm{L}$ PBS daily from 3 days prior to irradiation until the endpoint of the experiments. Six doses of $200 \mu \mathrm{g}$ anti-mouse PD-1 antibody (RMP1-14, BioXCell) or isotype (2A3, BioXCell) were administered i.p. in $100 \mu \mathrm{L}$ PBS on days 1, 3, 5, 7, 10,13 after irradiation.

Flow cytometry. Mouse tumors were excised and cut into $2-3 \mathrm{~mm}$ pieces to create single-cell suspensions. CT26.CL25 and B16-F10 tumors were dissociated with collagenase/hyaluronidase (STEMCELL Technologies, 07912) and murine tumor dissociation kit (Miltenyi, 130-096-730), respectively, following the manufacturers' recommendation. Dissected LNs were mechanically disrupted to create single cells and washed once using flow running buffer (0.1\% bovine serum albumin in PBS). All segregated cells were filtered using a $70 \mu \mathrm{m}$ nylon cell strainer (Corning).

Resulted single cells were incubated on ice with anti-CD16/32 antibodies for 15 min. The cells were stained using Live/Dead Fixable Violet or Aqua dyes (Thermo Fisher) and surface-labeled with anti-CD8 a BUV395 (clone 53-6.7; BD), anti-CD4 BV786 (clone GK1.5; BD), anti-CD3 BV786 or BV605 (clone 145-2C11; BD), anti-CD45 FITC or BV605 (clone 30-F11; BD), anti-PD-1 BV605 (clone J43; BD), anti-CTLA-4 PerCP/Cy5.5 (clone UC10-4B9; BioLegend), anti-Tim3 PE (clone B8.2C12; BioLegend), anti-LAG3 APC (clone C9B7W; BD), anti-CXCR3 BV650 (clone CXCR3-173; BioLegend), anti-CD11b BUV395 (clone M1/70, BD), antiF4/80 Ax647 (clone T45-2342; BD), and anti-Ly6G BV711 (clone 1A8; BD) for $30 \mathrm{~min}$ at room temperature (see Supplementary Table 1 and Supplementary Fig. 8).

For intracellular staining, cells were stimulated with cell activation cocktail containing phorbol myristate acetate (PMA)/ionomycin and Brefeldin A at $37^{\circ} \mathrm{C}$ for $4 \mathrm{~h}$ prior to surface-staining as described above. The cells were fixed and permeabilized using the forkhead box P3 (FoxP3)/Transcription Factor Staining Buffer Set (eBiosciences) according to the manufacturer's recommendation. Cells were stained with anti-FoxP3 PE (clone MF23; BD), anti-T-bet Ax647 (clone 4B10; BD), anti-IFN- $\gamma$ APC (clone XMG1.2; eBioscience), anti-TNF $\alpha$ PerCP/Cy5.5 (clone MP6-XT22; BioLegend), or anti-granzyme B Ax647 (clone GB11; BioLegend). Detailed information pertaining to antibodies and panel used can be found in Supplementary Table 1 and Supplementary Fig. 8.

All events were acquired immediately following sample processing using an LSR Fortessa flow cytometer (BD). All flow cytometric data analysis was performed using FCS Express 6 software package (De Novo Software).

Multiplex. To determine cytokine protein levels, serum samples from the experimental animals were analyzed using Millipore MCYTOMAG-70K Panel according to manufacturer's instructions. The plates were run on a Luminex 200 machine and the data analyzed using Upstate BeadView software.

NanoString. In brief, RNA was isolated from sorted CD8 ${ }^{+} \mathrm{T}$ cells of irradiated tumors and non-irradiated tumors in CT26 tumor-bearing WT or $\beta 2$-AR KO mice treated with radiation, using the RNeasy Plus Mini kit (QIAGEN). NanoString analysis was performed with the nCounter Analysis System at NanoString Technologies. The nCounter Mouse Immunology kit was used including 561 immunology-related mouse genes. All data are presented as $\log _{2}$ (fold change of WT/KO) with GraphPad Prism.

In vivo BLI. On Day 33 post tumor implantation, after surgical removal of the primary tumors, mice were administered $200 \mu \mathrm{L}$ D-luciferin $(150 \mathrm{mg} / \mathrm{kg}$; Caliper Life Sciences) by i.p. and then anaesthetized with isoflurane inhalation prior to imaging. Bioluminescent images of luciferase activity were acquired using BLI system (IVIS Spectrum, PerkinElmer) and analyzed with Living Image 4.3.1 software.

Histologic analysis of lung metastasis. The lung tissues were fixed in $10 \%$ formalin and embedded in paraffin and then stained with hematoxylin/eosin. The number of metastatic lesion was counted by light microscopy.
Statistical analysis. Student's $t$-test was used to compare data between two groups and tumor growth statistics were calculated using two-way ANOVA with Tukey analysis using GraphPad Prism. One-way ANOVA with Tukey posthoc tests was used to compare data between multiple groups. Differences were considered as statistical significance at $P<0.05$. All data are presented as mean \pm SEM

\section{Data availability}

All relevant data are presented in the article and Supplementary Information. The source data underlying Figs. 1-5 and Supplementary Figs. 1, 3-7 are provided as a Source Data file. All Nanostring data that support the findings of this study have been deposited in the National Center for Biotechnology Information Gene Expression Omnibus (GEO) and are accessible through the GEO Series accession number GSE145262.

Received: 12 June 2019; Accepted: 18 March 2020; Published online: 14 April 2020

\section{References}

1. Baskar, R., Dai, J., Wenlong, N., Yeo, R. \& Yeoh, K. W. Biological response of cancer cells to radiation treatment. Front. Mol. Biosci. 1, 24 (2014).

2. Abuodeh, Y., Venkat, P. \& Kim, S. Systematic review of case reports on the abscopal effect. Curr. Probl. Cancer 40, 25-37 (2016).

3. Siva, S., MacManus, M. P., Martin, R. F. \& Martin, O. A. Abscopal effects of radiation therapy: a clinical review for the radiobiologist. Cancer Lett. 356, 82-90 (2015).

4. Brix, N., Tiefenthaller, A., Anders, H., Belka, C. \& Lauber, K. Abscopal, immunological effects of radiotherapy: narrowing the gap between clinical and preclinical experiences. Immunol. Rev. 280, 249-279 (2017).

5. Demaria, S. et al. Ionizing radiation inhibition of distant untreated tumors (abscopal effect) is immune mediated. Int. J. Radiat. Oncol. Biol. Phys. 58, 862-870 (2004).

6. Demaria, S., Coleman, C. N. \& Formenti, S. C. Radiotherapy: changing the game in immunotherapy. Trends Cancer 2, 286-294 (2016).

7. Lesueur, P. et al. Review of the mechanisms involved in the abscopal effect and future directions with a focus on thymic carcinoma. Tumori 103, 217-222 (2017).

8. Formenti, S. C. \& Demaria, S. Systemic effects of local radiotherapy. Lancet Oncol. 10, 718-726 (2009).

9. Stone, H. B., Peters, L. J. \& Milas, L. Effect of host immune capability on radiocurability and subsequent transplantability of a murine fibrosarcoma. $J$. Natl Cancer Inst. 63, 1229-1235 (1979).

10. Demaria, S. et al. Immune-mediated inhibition of metastases after treatment with local radiation and CTLA-4 blockade in a mouse model of breast cancer. Clin. Cancer Res. 11, 728-734 (2005).

11. Formenti, S. C. \& Demaria, S. Combining radiotherapy and cancer immunotherapy: a paradigm shift. J. Natl Cancer Inst. 105, 256-265 (2013).

12. Golden, E. B., Demaria, S., Schiff, P. B., Chachoua, A. \& Formenti, S. C. An abscopal response to radiation and ipilimumab in a patient with metastatic non-small cell lung cancer. Cancer Immunol. Res. 1, 365-372 (2013).

13. Reynders, K., Illidge, T., Siva, S., Chang, J. Y. \& De Ruysscher, D. The abscopal effect of local radiotherapy: using immunotherapy to make a rare event clinically relevant. Cancer Treat. Rev. 41, 503-510 (2015).

14. Ngwa, W. et al. Using immunotherapy to boost the abscopal effect. Nat. Rev Cancer 18, 313-322 (2018).

15. Kang, J., Demaria, S. \& Formenti, S. Current clinical trials testing the combination of immunotherapy with radiotherapy. J. Immunother. Cancer $\mathbf{4}$, 51 (2016)

16. Vatner, R. E., Cooper, B. T., Vanpouille-Box, C., Demaria, S. \& Formenti, S. C. Combinations of immunotherapy and radiation in cancer therapy. Front. Oncol. 4, 325 (2014).

17. Crittenden, M. et al. Current clinical trials testing combinations of immunotherapy and radiation. Semin. Radiat. Oncol. 25, 54-64 (2015).

18. Spiotto, M., Fu, Y. X. \& Weichselbaum, R. R. The intersection of radiotherapy and immunotherapy: mechanisms and clinical implications. Sci. Immunol. 1, eaag1266 (2016)

19. Cirincione, R. et al. High-intensity focused ultrasound- and radiation therapyinduced immuno-modulation: comparison and potential opportunities. Ultrasound Med. Biol. 43, 398-411 (2017).

20. Khan, M. K., Nasti, T. H., Buchwald, Z. S., Weichselbaum, R. R. \& Kron, S. J. Repurposing drugs for cancer radiotherapy: early successes and emerging opportunities. Cancer J. 25, 106-115 (2019).

21. Whiteside, T. L., Demaria, S., Rodriguez-Ruiz, M. E., Zarour, H. M. \& Melero, I. Emerging opportunities and challenges in cancer immunotherapy. Clin. Cancer Res. 22, 1845-1855 (2016) 
22. Eng, J. W. et al. Housing temperature-induced stress drives therapeutic resistance in murine tumour models through beta2-adrenergic receptor activation. Nat. Commun. 6, 6426 (2015).

23. Cannon, B. \& Nedergaard, J. Brown adipose tissue: function and physiological significance. Physiol. Rev. 84, 277-359 (2004).

24. Hylander, B. L., Gordon, C. J. \& Repasky, E. A. Manipulation of ambient housing temperature to study the impact of chronic stress on immunity and cancer in mice. J. Immunol. 202, 631-636 (2019).

25. Hylander, B. L. \& Repasky, E. A. Thermoneutrality, mice, and cancer: a heated opinion. Trends Cancer 2, 166-175 (2016).

26. Hankenson, F. C., Marx, J. O., Gordon, C. J. \& David, J. M. Effects of rodent thermoregulation on animal models in the research environment. Comp. Med. 68, 425-438 (2018).

27. Gordon, C. J. The mouse thermoregulatory system: Its impact on translating biomedical data to humans. Physiol. Behav. 179, 55-66 (2017).

28. Kokolus, K. M. et al. Baseline tumor growth and immune control in laboratory mice are significantly influenced by subthermoneutral housing temperature. Proc. Natl Acad. Sci. USA 110, 20176-20181 (2013).

29. Bucsek, M. J. et al. beta-Adrenergic signaling in mice housed at standard temperatures suppresses an effector phenotype in CD8(+) T cells and undermines checkpoint inhibitor therapy. Cancer Res. 77, 5639-5651 (2017).

30. Mohammadpour, $\mathrm{H}$. et al. Blockade of host beta2-adrenergic receptor enhances graft-versus-tumor effect through modulating APCs. J. Immunol. 200, 2479-2488 (2018).

31. Leigh, N. D. et al. Housing temperature-induced stress is suppressing murine graft-versus-host disease through beta2-adrenergic receptor signaling. J. Immunol. 195, 5045-5054 (2015).

32. Mohammadpour, $\mathrm{H}$. et al. beta 2 adrenergic receptor-mediated signaling regulates the immunosuppressive potential of myeloid-derived suppressor cells. J. Clin. Invest. 129, 5537-5552 (2019)

33. Qiao, G. et al. Beta-Adrenergic signaling blocks murine CD8(+) T-cell metabolic reprogramming during activation: a mechanism for immunosuppression by adrenergic stress. Cancer Immunol. Immunother. 68, 11-22 (2019)

34. Hylander, B. L., Eng, J. W. \& Repasky, E. A. The impact of housing temperature-induced chronic stress on preclinical mouse tumor models and therapeutic responses: an important role for the nervous system. $A d v$. Exp. Med. Biol. 1036, 173-189 (2017).

35. Cole, S. W. \& Sood, A. K. Molecular pathways: beta-adrenergic signaling in cancer. Clin. Cancer Res. 18, 1201-1206 (2012).

36. Cole, S. W., Nagaraja, A. S., Lutgendorf, S. K., Green, P. A. \& Sood, A. K. Sympathetic nervous system regulation of the tumour microenvironment. Nat. Rev. Cancer 15, 563-572 (2015).

37. MacDonald, C. R. et al. Adrenergic receptor signaling regulates the response of tumors to ionizing radiation. Radiat. Res. 191, 585-589 (2019).

38. Rodriguez-Ruiz, M. E., Vanpouille-Box, C., Melero, I., Formenti, S. C. \& Demaria, S. Immunological mechanisms responsible for radiation-induced abscopal effect. Trends Immunol. 39, 644-655 (2018).

39. Kozin, S. V. et al. Recruitment of myeloid but not endothelial precursor cells facilitates tumor regrowth after local irradiation. Cancer Res. 70, 5679-5685 (2010).

40. Shiao, S. L. et al. TH2-polarized CD4(+) T cells and macrophages limit efficacy of radiotherapy. Cancer Immunol. Res. 3, 518-525 (2015).

41. Lin, Y., Xu, J. \& Lan, H. Tumor-associated macrophages in tumor metastasis: biological roles and clinical therapeutic applications. J. Hematol. Oncol. 12, 76 (2019).

42. Peske, J. D., Woods, A. B. \& Engelhard, V. H. Control of CD8 T-Cell infiltration into tumors by vasculature and microenvironment. Adv. Cancer Res. 128, 263-307 (2015).

43. Griffith, J. W., Sokol, C. L. \& Luster, A. D. Chemokines and chemokine receptors: positioning cells for host defense and immunity. Annu. Rev. Immunol. 32, 659-702 (2014).

44. Mikucki, M. E. et al. Unlocking tumor vascular barriers with CXCR3 implications for cancer immunotherapy. Oncoimmunology 5, e1116675 (2016).

45. Demaria, S. \& Formenti, S. C. Role of T lymphocytes in tumor response to radiotherapy. Front. Oncol. 2, 95 (2012).

46. Nakai, A., Hayano, Y., Furuta, F., Noda, M. \& Suzuki, K. Control of lymphocyte egress from lymph nodes through beta2-adrenergic receptors. $J$. Exp. Med. 211, 2583-2598 (2014).

47. Nakai, A. \& Suzuki, K. Adrenergic control of lymphocyte trafficking and adaptive immune responses. Neurochem. Int. 130, 104320 (2019).

48. Aoki, M., Aoki, H., Ramanathan, R., Hait, N. C. \& Takabe, K. Sphingosine-1phosphate signaling in immune cells and inflammation: roles and therapeutic potential. Mediators Inflamm. 2016, 8606878 (2016).

49. Baer, A., Colon-Moran, W. \& Bhattarai, N. Characterization of the effects of immunomodulatory drug fingolimod (FTY720) on human T cell receptor signaling pathways. Sci. Rep. 8, 10910 (2018).
50. Schwab, S. R. \& Cyster, J. G. Finding a way out: lymphocyte egress from lymphoid organs. Nat. Immunol. 8, 1295-1301 (2007).

51. Pulaski, B. A. \& Ostrand-Rosenberg, S. Mouse 4T1 breast tumor model. Curr. Protoc. Immunol. Chapter 20 Unit 20, 22 (2001).

52. Graham, K. \& Unger, E. Overcoming tumor hypoxia as a barrier to radiotherapy, chemotherapy and immunotherapy in cancer treatment. Int. J. Nanomed. 13, 6049-6058 (2018).

53. Tang, L. et al. Role of metabolism in cancer cell radioresistance and radiosensitization methods. J. Exp. Clin. Cancer Res. 37, 87 (2018).

54. Moeller, B. J., Richardson, R. A. \& Dewhirst, M. W. Hypoxia and radiotherapy: opportunities for improved outcomes in cancer treatment. Cancer Metastasis Rev. 26, 241-248 (2007).

55. Mikucki, M. E. et al. Non-redundant requirement for CXCR3 signalling during tumoricidal T-cell trafficking across tumour vascular checkpoints. Nat. Commun. 6, 7458 (2015).

56. Qin, J. F. et al. Adrenergic receptor beta2 activation by stress promotes breast cancer progression through macrophages M2 polarization in tumor microenvironment. BMB Rep. 48, 295-300 (2015).

57. $\mathrm{Wu}, \mathrm{L}$. et al. Bidirectional role of beta2-adrenergic receptor in autoimmune diseases. Front. Pharm. 9, 1313 (2018).

58. Kokolus, K. M. et al. Beta blocker use correlates with better overall survival in metastatic melanoma patients and improves the efficacy of immunotherapies in mice. Oncoimmunology 7, e1405205 (2018)

59. Lugade, A. A. et al. Local radiation therapy of B16 melanoma tumors increases the generation of tumor antigen-specific effector cells that traffic to the tumor. J. Immunol. 174, 7516-7523 (2005).

60. Gameiro, S. R. et al. Tumor cells surviving exposure to proton or photon radiation share a common immunogenic modulation signature, rendering them more sensitive to T cell-mediated killing. Int. J. Radiat. Oncol. Biol. Phys. 95, 120-130 (2016)

61. Buchwald, Z. S. et al. Radiation, immune checkpoint blockade and the abscopal effect: a critical review on timing, dose and fractionation. Front. Oncol. 8, 612 (2018).

62. Singh, A. K. et al. A pilot study of stereotactic body radiation therapy combined with cytoreductive nephrectomy for metastatic renal cell carcinoma. Clin. Cancer Res. 23, 5055-5065 (2017).

63. Britschgi, C., Riesterer, O., Burger, I. A., Guckenberger, M. \& CurioniFontecedro, A. Report of an abscopal effect induced by stereotactic body radiotherapy and nivolumab in a patient with metastatic non-small cell lung cancer. Radiat. Oncol. 13, 102 (2018).

64. Komatsu, T., Nakamura, K. \& Kawase, A. Abscopal effect of nivolumab in a patient with primary lung cancer. J. Thorac. Oncol. 12, e143-e144 (2017).

65. Tsui, J. M., Mihalcioiu, C. \& Cury, F. L. Abscopal effect in a stage IV melanoma patient who progressed on pembrolizumab. Cureus 10, e2238 (2018).

66. Zhao, X., Kang, J. \& Zhao, R. Abscopal effect of radiation on lymph node metastasis in esophageal carcinoma: a case report and literature review. Oncol. Lett. 16, 3555-3560 (2018).

67. Postow, M. A. et al. Immunologic correlates of the abscopal effect in a patient with melanoma. N. Engl. J. Med. 366, 925-931 (2012)

68. Shi, F., Wang, X., Teng, F., Kong, L. \& Yu, J. Abscopal effect of metastatic pancreatic cancer after local radiotherapy and granulocyte-macrophage colony-stimulating factor therapy. Cancer Biol. Ther. 18, 137-141 (2017).

69. Chaudhary, K. R. et al. Effects of beta-adrenergic antagonists on chemoradiation therapy for locally advanced non-small cell lung cancer. J. Clin. Med. 8, 575 (2019).

70. Wang, H. M. et al. Improved survival outcomes with the incidental use of beta-blockers among patients with non-small-cell lung cancer treated with definitive radiation therapy. Ann. Oncol. 24, 1312-1319 (2013).

\section{Acknowledgements}

The authors would like to thank Cameron MacDonald for his help in several aspects of this project and review of the data. This work was supported by NIH grants (R01 CA236390, R01 CA099326, R01 CA205246 to E.A.R. and F32CA239356 to H.M.); The Roswell Park Alliance Foundation; The National Cancer Institute (NCI) grant P30CA016056 and shared instrumentation grant (S10OD16450) involving the use of Roswell Park Comprehensive Cancer Center's Flow and Image Cytometry, Immune Analysis Shared Resources and Translational Imaging Shared Resource.

\section{Author contributions}

M.C. and E.A.R. conceived of and designed the project with assistance from X.-Y.W., J.R S. and A.K.S.; M.C. performed the majority of the experiments and analyzed data with the help of G.Q. and H.M.; M.C., B.L.H. and E.A.R. wrote and all authors edited the manuscript. E.A.R. supervised the project.

\section{Competing interests}

The authors declare no competing interests. 


\section{Additional information}

Supplementary information is available for this paper at https://doi.org/10.1038/s41467020-15676-0

Correspondence and requests for materials should be addressed to E.A.R.

Peer review information Nature Communications thanks Mark Dowhirst and the other, anonymous, reviewer(s) for their contribution to the peer review of this work.

Reprints and permission information is available at http://www.nature.com/reprints

Publisher's note Springer Nature remains neutral with regard to jurisdictional claims in published maps and institutional affiliations. (c) (i) Open Access This article is licensed under a Creative Commons Attribution 4.0 International License, which permits use, sharing, adaptation, distribution and reproduction in any medium or format, as long as you give appropriate credit to the original author(s) and the source, provide a link to the Creative Commons license, and indicate if changes were made. The images or other third party material in this article are included in the article's Creative Commons license, unless indicated otherwise in a credit line to the material. If material is not included in the article's Creative Commons license and your intended use is not permitted by statutory regulation or exceeds the permitted use, you will need to obtain permission directly from the copyright holder. To view a copy of this license, visit http://creativecommons.org/ licenses/by/4.0/.

(C) The Author(s) 2020 